\title{
Electro-Absorption Modulator vertically integrated on a VCSEL: microstrip-based high-speed electrical injection on top of a BCB layer
}

\author{
Ludovic Marigo-Lombart, Christophe Viallon, Alexandre Rumeau, Alexandre Arnoult, Aurélie Lecestre, \\ Laurent Mazenq, Ayad Ghannam, Hugo Thienpont, Krassimir Panajotov, and Guilhem Almuneau
}

\begin{abstract}
Vertical-Cavity Surface-Emitting Lasers with vertically-integrated electro-absorption modulators (EAMVCSELs) potentially can reach higher modulation bandwidths than directly current-modulated VCSELs. The aforementioned device modulation capabilities are however currently restricted by their electrical contact parasitics. It thus becomes critical to optimize their access line to improve performance. In this paper, we numerically and experimentally demonstrate that a microstrip (MS) access line using a planarized BCB layer exhibits improved high-frequency characteristics compared to the coplanar waveguide (CPW) access line used to-date since it reduces the losses induced by the doped substrates. We also use this opportunity to introduce an innovative technique for BCB-layer planarization which is not only compatible with the EAM-VCSEL double-mesa structure but is also independent of the device pitch. The resulting BCB layer dielectric permittivity and losses are measured up to $100 \mathrm{GHz}$ and a side-by-side comparison of the electrical response of three-electrodes MS and CPW access lines is subsequently carried out. Finally, using the measured pad and access line RF responses and the EAM modulation characteristics, the devices with MS access are shown to be no longer limited by their electrode parasitics but by the modulator internal impedance.
\end{abstract}

Index Terms-High-speed modulation, Electro-absorption modulator, VCSEL, BCB.

\section{INTRODUCTION}

$\mathbf{I}$ $\mathrm{N}$ order to increase the modulation bandwidth, the vertical integration of an electro-optic modulator onto a VCSEL (EOM-VCSEL) has already been demonstrated to be a competitive approach [1]-[4]. In that case, a double mesa structure is required with three contacts: one on the top for applying a voltage to the EOM, one middle contact for a shared ground, and one at the bottom for the VCSEL CW current injection.

This approach requires : 1) planarization with a low permittivity layer to decrease the parasitic capacitances; 2) characterization of dielectric properties of such a layer up to frequencies as high as $100 \mathrm{GHz}$; 3) development of a MS access line to

L. Marigo-Lombart, C. Viallon, A. Rumeau, A. Arnoult, A. Lecestre, L. Mazenq and G. Almuneau are with LAAS-CNRS, Université de Toulouse, CNRS, UPS, Toulouse, France, e-mail: almuneau@laas.fr.

A. Ghannam is with 3DiS Technologies, Labège 31676, France.

H. Thienpont and K. Panajotov are with the Department of Applied Physics and Photonics (TW-TONA), Vrije Universiteit Brussel, Pleinlaan 2, B-1050 Brussels, Belgium.

The authors gratefully acknowledge the technological support of RENATECH (French Network of Technology Platforms) within LAAS-CNRS cleanroom infrastructure and the financial support by the Methusalem foundation, Belgium.

Manuscript received xxxxx; revised xxxx. avoid the losses induced by the electromagnetic (EM) coupling of the signal with the doped substrate. In this paper, we address all the above issues in the case of an EAM-VCSEL, in which a voltage-driven electro-absorber placed above modulates the output light of the steady-biased VCSEL.

Indeed, all the groups that have already worked on vertical integration of an EOM onto a VCSEL used highly doped substrate for the current injection in the VCSEL without considering, to our knowledge, the induced EM losses for the modulator. In [5], an optical modulation bandwidth exceeding $35 \mathrm{GHz}$ was demonstrated using p-doped substrate, intracavity injection scheme for the modulator and CPW access line. Chen et al [6] worked with a Ground-Signal-Signal-Ground (GSSG) access line that presents the advantage of providing all the access on the same level. However, the substrate doping remains an issue and the fabrication process complexity is considerably increased. Finally, Yakimov et al. [4] used a doped substrate with a CPW access line. Although a p-doped substrate has been chosen for the EAM-VCSEL presented in this paper, we show that an undoped substrate could potentially improve the performance of a CPW-based device at the cost of a more complex process for VCSEL fabrication that will not be discussed here.

In order to increase the EOM electrical frequency bandwidth, a low-permittivity material is required for separating the modulator electrode pads. To this aim, metal air bridge can be used but this approach suffers from a complicated process flow. A thick dielectric layer can also be implemented but it is difficult to shape it by photolithography and etching while ensuring precise pattern alignment. Finally, polyimides are very good candidates and are most often used to overcome these last issues. Compared to $\mathrm{SiO}_{x}$ or $\mathrm{Si}_{x} \mathrm{~N}_{y}$, polyimides present lower dielectric constant [7]. We develop in this paper a novel approach to planarize Bis-benzocyclobutene (BCB) on top of semiconductor devices by using a mechanical press benefiting from a nanoimprint tool to apply a high pressure and temperature and spread the $\mathrm{BCB}$ and improve its flatness. BCB is nowadays of high interest in many applications such as wafer bonding, 3D interconnect processes or high-speed devices in MEMS, silicon and photonics applications. Indeed, it presents good mechanical properties after hard cure, low moisture and low permittivity $\left(\epsilon_{r}\right)$. Furthermore, BCB is more easily definable by spin-coating and photolithography compared to dielectric layers that require plasma or chemical etching, which can significantly damage the semiconductor 
topmost layers. These $\mathrm{BCB}$ properties, despite its relatively low thermal conductivity, make it very attractive for microwave optoelectronics. Indeed, BCB is extensively used in all high speed semiconductor based optical devices such as Distributed Feedback Lasers, Vertical-Cavity Surface-Emitting Lasers (VCSEL) and electro-optical modulators.

The precise knowledge of $\mathrm{BCB}$ permittivity and losses for frequencies up to $100 \mathrm{GHz}$ is crucial for predicting and optimizing the influence of the RF electrical access line on device performance. The dielectric constant of BCB provided by the manufacturer is 2.65 at $1 \mathrm{MHz}$ and 2.5 in the $1-10 \mathrm{GHz}$ frequency range, and within a temperature range from 30 to $200{ }^{\circ} \mathrm{C}$, whatever the BCB solution [8]. The few charaterization results available from the literature report $\epsilon_{r}$ values in the range of 2.5-2.7, depending on the measurement technique and the used BCB solution [9]-[13]. Furthermore, BCB has been mostly characterized at frequencies below $40 \mathrm{GHz}$. In [10], a value of 2.7 has been extracted up to $60 \mathrm{GHz}$ by fitting the Sparameters of a $50 \Omega$ MS line involving a $26 \mu \mathrm{m}$-thick BCB layer. In [12], 2.65 was measured in the range $11-65 \mathrm{GHz}$ from a CPW. In this paper, the BCB permittivity and the losses are characterized up to $100 \mathrm{GHz}$.

In order to provide carrier injection in VCSELs, a CPW access line is most commonly used [14], [15]. To avoid EM coupling with the highly doped bottom n-contact layer close to the active region, the n-layer is etched away under the pad line which constitutes an additional process step. However, this approach is not possible for EAM-VCSEL, in which a highly doped substrate must be used as a third electrode. Therefore, we develop in this paper an optimized MS access line. We study the influence of the substrate doping and compare the high frequency properties of the MS line configuration to that of CPW access line on N, P and Not Intentionally Doped (NID) substrates with several test structures.

\section{BCB INNOVATIVE PLANARIZATION PROCESS}

\section{A. BCB technological implementation}

As described in [16], BCB can be simply spin-coated with as good degree of planarization (DOP) as $95 \%$. This optimum DOP is achieved for device pitch of about 400 $500 \mu \mathrm{m}$ but it worsens when the distance between components decreases down to $150-200 \mu \mathrm{m}$, which is commonly used for VCSEL manufacturing. The double mesa structure of the EAM-VCSEL is also a major hurdle to get the aimed surface flatness. Furthermore, the BCB process requires an additional photolithography step with a tight alignment in order to open an access down to the semiconductor surface.

To overcome all these issues, it is possible to planarize the BCB at the height level of the mesa structure. To do so, chemical mechanical polishing (CMP) can be used to lower the BCB thickness after thermal curing but this process is difficult and may lead to unsatisfying results [17] and possible contamination. Alternatively, one can etch the BCB in a controlled way by plasma reactive etching. However, in the case of non-perfect $\mathrm{BCB}$ planarization, a slight swelling is created after spin-coating above the mesa edge and is replicated after dry etching. This can cause a short-circuit as shown in the topmost mesa corner.
Finally, a mechanical pressing techniques using nanoimprint tool can be advantageously and reliably used for polymer planarization as previously demonstrated in [18] and [19]. After obtaining a sufficient BCB flatness, Yang et al. [20] reported a study on reactive ion etching (RIE) conditions for $\mathrm{BCB}$ etching with $\mathrm{SF}_{6}$-based gas mixtures. A stop-etch layer (for example, $\mathrm{Si}_{3} \mathrm{~N}_{4}$ ) can be used to accurately match the aimed etch depth [21].

\section{B. Self-aligned BCB planarization process}

We present in this section an innovative process combining a step of mechanical pressing in order to flatten the BCB surface with a step of reproducible dry etching of the BCB excess film, while keeping the surface smooth enough for high-frequency low-loss injection. Prior to the $\mathrm{BCB}$ coating, a $\mathrm{SiO}_{x}$ layer is deposited by inductively coupled plasma chemical vapour deposition (ICPCVD) to improve BCB adhesion [22]. After a spin-coating with ramping-up speed and a soft bake of $\mathrm{BCB}$ 4026-46 resist, a layer thickness of $10.6 \mu \mathrm{m}$ is obtained. After the soft-bake, done at $80^{\circ} \mathrm{C}$, it is still possible to reflow the BCB layer by heating it to temperature of $100^{\circ} \mathrm{C}$ for $6 \mathrm{~min}$ utes. We also demonstrate that neither higher temperature nor longer heating are necessary. Taking advantage of this reflow, a mechanical press is used, with a nano-imprint equipment under $1.72 \mathrm{MPa}$ and $100{ }^{\circ} \mathrm{C}$ for 6 minutes in order to perfectly flatten the BCB layer.

After the planarization and before the dry etch, it is mandatory to anneal the BCB by hardcure treatment at $250{ }^{\circ} \mathrm{C}$ for one hour. In such a way most of the contained solvent is removed and the $\mathrm{BCB}$ mechanical strength and electrical properties are improved.

The final step of the BCB planarization is the thickness readjustment in order to strictly outcrop the mesa heights, thus resulting in a perfect planarized configuration whatever the spacing between etched mesas. This etching step is applied on the overall surface of the sample without any masking materials. Different conditions have been tested for the dry etching of BCB layer with various gases, pressure and, ICP and RF powers. By inductively coupled plasma reactive ion etching (ICP-RIE), the lowest roughness with controllable etching rate is achieved with a $\mathrm{O}_{2} / \mathrm{SF}_{6}$ ratio of $98 / 2$ at $80 \mathrm{mTorr}$ and ICP and bias powers of $500 \mathrm{~W}$ and $40 \mathrm{~W}$, respectively. More details on this process can be found in [22].

The BCB surface roughness directly influences the quality of the Au top contact and so the high-frequency performance. A slight roughness is however required to ensure a good adhesion of the metal on the BCB, and is naturally created by the dry etch process. We monitor in-situ the reflected $670 \mathrm{~nm}$ laser signal: its average amplitude providing the etch depth, while the amplitude oscillations providing the etched surface roughness. AFM measurements show a roughness of only $10 \mathrm{~nm}$ on the metal layer deposited on partially etched BCB.

\section{BCB MICROWAVE CHARACTERIZATION}

Most commonly, the extraction of the dielectric microwave characteristics of insulating materials is done by inserting the studied material into a waveguide or a transverse electric and 
magnetic (TEM) transmission line. The complex dielectric constant and permeability are then retrieved from the measured scattering matrix as described in [23].

In our case, only a thin layer of BCB can be deposited onto a substrate of different nature, which makes it virtually impossible to design a purely TEM structure. We therefore chose to focus on the quasi-TEM MS transmission line for its ease of fabrication: the BCB layer is placed between the signal path and the lower ground plane. Since the EM wave propagates partly in the air and partly in the material to be characterized, measurements give only access to an effective permittivity. The proven analytical models that are available for the case of the MS line are then used to retrieve the relative dielectric permittivity from the measured effective one.

\section{A. Wafer fabrication}

Only a few steps are required to fabricate MS line structures for $\mathrm{BCB}$ characterization. First, a ground plane located under the BCB is deposited on the whole sample. Then, a BCB layer is spin-coated and an additional photolithography step is employed to form openings used to electrically connect the ground plane to the ground pads located on the top of the BCB layer. Finally, after a heating treatment, a lift-off is performed to fabricate the RF pads, the vias and the transmission line.

In the case of an EAM vertically integrated onto a VCSEL, the first mesa is $3 \mu \mathrm{m}$ thick and another etch step is required to reach the active region of the VCSEL and to provide lateral electrical and optical confinement. That corresponds to a global mesa thickness of $6 \mu \mathrm{m}$.

Several MS lines with lengths of 200, 300, 700, $1200 \mu \mathrm{m}$ and a $14 \mu \mathrm{m}$-width signal track have been drawn, as done in [24]. These lines present a characteristic impedance of $53 \Omega$ using Cyclotene ${ }^{\mathrm{TM}}$ manufacturer standard permittivity of 2.65 . Coplanar squared RF pads with $60 \mu \mathrm{m}$ widths are added at the MS lines terminals to enable Ground-Signal-Ground (GSG) RF probe contacts.

\section{B. Access pads de-embedding}

The first step prior to the extraction of $\mathrm{BCB}$ dielectric characteristics is to remove the RF pads contribution from raw measured data. Usually, an electrical equivalent lumped model of the pad is retrieved from dedicated Short, Open and/or Thru dummies structures [25]. Open and Short patterns are used to calculate the equivalent parallel capacitance and series inductance of the pad, respectively. But these patterns are becoming problematic with increasing frequency due to the non-ideality of the corresponding terminations [26]. Such problems are avoided if the Open and Short devices are replaced by only one thru pattern but this technique introduces new issues [26], [27]. Indeed, since the pad is modeled by lumped elements, all these methods are valid only for access dimensions much smaller than the wavelength (typ. $\lambda_{g} / 20$ ) of the highest test frequency. A short thru pattern is hence desirable but the close proximity of the signal pads and the probes introduces a cross-talk that leads to an incorrect pad modeling [27]. The technique proposed here avoids all these issues. Two transmission lines of different lengths are required instead of Open/Short or Thru dummies structures. The method described below borrows an important part of [28] but differs from it by the assumptions used to simplify the problem to make it consistent and by the subsequent nature of the model chosen for the pad. In [28], the access pads are modeled by a distributed reciprocal and symmetric $\left(S_{11}=S_{22}\right)$ two-port network. Here, the symmetric condition is impaired because of the large step in the widths between the pad to the MS line that leads to $S_{11} \neq S_{22}$, especially at high frequencies. Removing this symmetry leads to an under-determined problem that is handled by re-introducing a simplified lumped pad model similar to the one used in the Open/Short or Thru de-embedding methods.

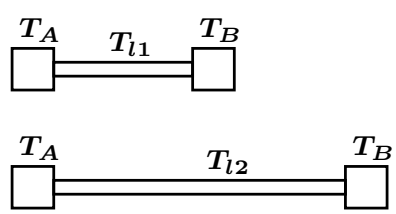

(a)

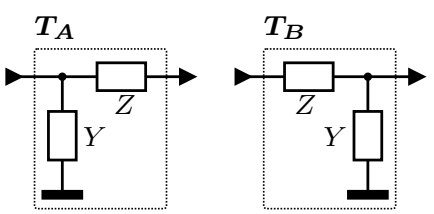

(b)
Fig. 1. (a) Transmission lines $L_{1}$ and $L_{2}$ with related chain matrices. (b) Lumped models of pads A and B used to calculate $\boldsymbol{T}_{\boldsymbol{A}}$ and $\boldsymbol{T}_{\boldsymbol{B}}$.

The two lines $L_{1}$ and $L_{2}$ and the terminating pads are shown in Fig. 1a. If the ABCD formalism is used, the measured scattering parameters of lines $L_{1}$ and $L_{2}$ are converted into chain parameters, marked $\boldsymbol{M}_{1}$ and $\boldsymbol{M}_{2}$, respectively

$$
\boldsymbol{M}_{\mathbf{1}}=\left(\begin{array}{ll}
m_{11} & m_{12} \\
m_{21} & m_{22}
\end{array}\right) \text { and } \boldsymbol{M}_{\mathbf{2}}=\left(\begin{array}{ll}
p_{11} & p_{12} \\
p_{21} & p_{22}
\end{array}\right) .
$$

Both transmission lines display the same characteristic impedance $Z_{c}$ and propagation constant $\gamma$ but differ by the lengths, written as $l_{1}$ and $l_{2}$, respectively. The surrounding signal pads are modeled by the lumped networks drawn in Fig. 1b. Using these parameters, $\boldsymbol{T}_{A}$ and $\boldsymbol{T}_{\boldsymbol{B}}, \boldsymbol{T}_{l 1}$ and $\boldsymbol{T}_{l 2}$ are expressed as follows

$$
\begin{gathered}
\boldsymbol{T}_{\boldsymbol{A}}=\left(\begin{array}{cc}
1 & Z \\
Y & Y Z+1
\end{array}\right), \boldsymbol{T}_{\boldsymbol{B}}=\left(\begin{array}{cc}
Y Z+1 & Z \\
Y & 1
\end{array}\right) \\
\boldsymbol{T}_{\boldsymbol{l} \mathbf{1}}=\left(\begin{array}{cc}
\cosh \left(\gamma l_{1}\right) & Z_{c} \sinh \left(\gamma l_{1}\right) \\
\frac{1}{Z_{c}} \sinh \left(\gamma l_{1}\right) & \cosh \left(\gamma l_{1}\right)
\end{array}\right) \\
\boldsymbol{T}_{\boldsymbol{l} \mathbf{2}}=\left(\begin{array}{cc}
\cosh \left(\gamma l_{2}\right) & Z_{c} \sinh \left(\gamma l_{2}\right) \\
\frac{1}{Z_{c}} \sinh \left(\gamma l_{2}\right) & \cosh \left(\gamma l_{2}\right)
\end{array}\right)
\end{gathered}
$$

From Fig. 1a, we write

$$
\begin{aligned}
& M_{1}=T_{A} T_{l 1} T_{B} \\
& M_{2}=T_{A} T_{l 2} T_{B} .
\end{aligned}
$$

Expressions (4) and (5) are re-writen as follows

$$
\begin{aligned}
& M_{\mathbf{1}} T_{B}^{-1}=T_{A} T_{l 1} \\
& M_{\mathbf{2}} T_{B}{ }^{-1}=T_{A} T_{l \mathbf{2}} .
\end{aligned}
$$

Since transmission lines are symmetric devices, $m_{11}=m_{22}$ and $p_{11}=p_{22}$ are observed experimentally. Using these relations and expanding (6) and (7), the following expressions are derived

$$
m_{12}=\left[\cosh \left(\gamma l_{1}\right)+m_{11}\right] \frac{Z}{1+Y Z}+\frac{Z_{c}}{1+Y Z} \sinh \left(\gamma l_{1}\right)
$$




$$
\begin{array}{r}
m_{21}=\left[\cosh \left(\gamma l_{1}\right)+m_{11}\right] Y+\frac{1+Y Z}{Z_{c}} \sinh \left(\gamma l_{1}\right) \\
p_{12}=\left[\cosh \left(\gamma l_{2}\right)+p_{11}\right] \frac{Z}{1+Y Z}+\frac{Z_{c}}{1+Y Z} \sinh \left(\gamma l_{2}\right) \\
p_{21}=\left[\cosh \left(\gamma l_{2}\right)+p_{11}\right] Y+\frac{1+Y Z}{Z_{c}} \sinh \left(\gamma l_{2}\right) .
\end{array}
$$

After defining the matrix $\boldsymbol{A}$ as

$$
\boldsymbol{A}=\left(\begin{array}{cc}
\cosh \left(\gamma l_{1}\right)+m_{11} & \sinh \left(\gamma l_{1}\right) \\
\cosh \left(\gamma l_{2}\right)+p_{11} & \sinh \left(\gamma l_{2}\right)
\end{array}\right)
$$

the expressions (8) to (11) are rewritten and combined in a matrix form using (12) to get the final equation

$$
\left(\begin{array}{cc}
Y & \frac{Z}{1+Y Z} \\
\frac{1+Y Z}{Z_{c}} & \frac{Z_{c}}{1+Y Z}
\end{array}\right)=A^{-1} \cdot\left(\begin{array}{cc}
m_{21} & m_{12} \\
p_{21} & p_{12}
\end{array}\right) .
$$

Equation (13) can be solved for $Y, Z$ and $Z_{c}$ knowing $M_{1}$ and $\boldsymbol{M}_{2}$ and the related propagation constant $\gamma \cdot \gamma$ can be extracted beforehand from $\boldsymbol{M}_{1}$ and $\boldsymbol{M}_{2}$, i.e. by using the method proposed in [29].

\section{Extraction of the electrical characteristics of $B C B$}

First, the complex effective permittivity $\overline{\epsilon_{e f f}}$ is calculated from the de-embedded measurement data of one of the two transmission lines. The transmission-reflection method introduced in [23] and modified in [24] is used to extract $\overline{\epsilon_{e f f}}$. The relative permittivity $\epsilon_{r}$, as well as the loss tangent term tan $\delta$ are then determined either by a direct computation carried out by using the analytic equations derived from Bahl's model (method 1) [30], or by using an iterative algorithm based on the Jensen-Hammerstad model (method 2) [31]. The choice between these two methods is dictated by the validity range of each model regarding the MS line geometry. It mainly depends on the thickness over height $t / h$ ratio of the analyzed MS line. These two techniques, as well as the form factor validity range have been investigated in [24]. Accurately measured geometric data of the line are also required to insure the best possible accuracy of the extraction of the BCB electrical characteristics.

The measured $S_{21}$ parameters of all MS lines are shown in Fig. 2a. A vector network analyser Anritsu Vectorstar, its Broadband Test Set 3738A and a probe station were employed to perform measurements up to $110 \mathrm{GHz} .150 \mu \mathrm{m}$ pitch Cascade Infinity GSG probes were also used.

The BCB permittivity $\epsilon_{r}$ and $\tan \delta$ extracted by using the two previously described methods reach the same outcome, as seen in Fig. 2b. At low frequency, a permittivity of 2.6 is measured. It decreases to 2.5 at $70 \mathrm{GHz}$. Past $70 \mathrm{GHz}$, we go beyond the validity range of the lumped element approximation used to model the pads, which probably induces the change of slope observed in the $\epsilon_{r}$ vs frequency curve. $\tan \delta$ is defined as the ratio between imaginary and real parts of the complex permittivity $\left(\epsilon^{\prime \prime} / \epsilon^{\prime}\right)$. Its values are comprised between 0.01 and 0.03 in the $0.05-70 \mathrm{GHz}$ range.

The method developed here to extract the BCB permittivity from MS lines measurements will be reused in Section V-B to calculate the access line contribution on the measured impedance of the modulator.
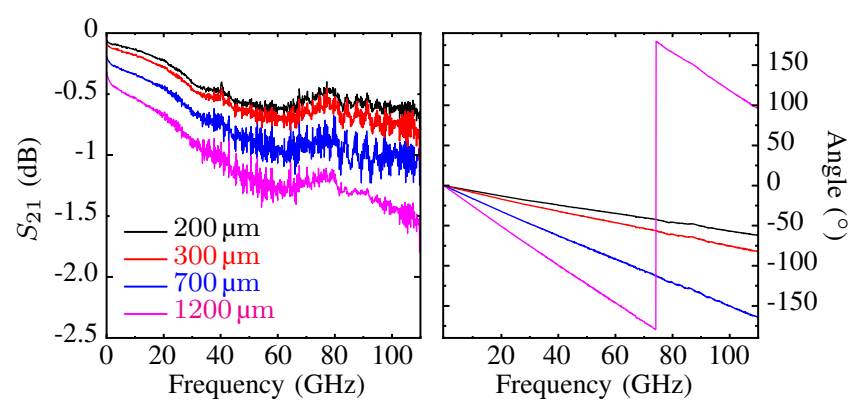

(a)

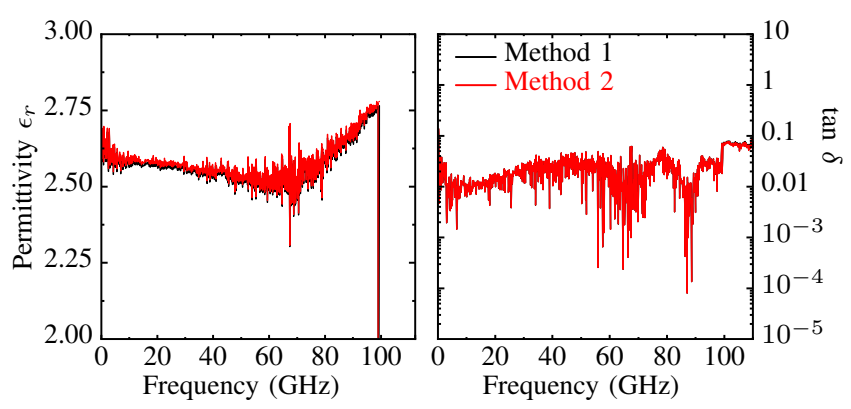

(b)

Fig. 2. (a) Measured transmission parameter of MS lines of 200 (black), 300 (red), 700 (blue) and $1200 \mu \mathrm{m}$ (magenta). (b) Extracted BCB data.

\section{HIGH-FREQUENCY INJECTION DESIGN}

The measured BCB dielectric data are now used to design the pads and the access line and to minimize the HF injection losses. In this section, two designs are compared: the CPW commonly used by the VCSEL community, and the MS access line proposed here.

\section{A. CPW access line}

1) Pad design: we based our first approach on a standard coplanar structure where both the ground plane and the access line are located on the top of the BCB layer as shown in Fig. 3a. The ground plane surrounds the signal pad to promote ground equipotentiality and avoid slot-line propagation mode. For clarity, only heights are on scale, lateral dimensions have been shortened. Here, the signal pad is tapered to decrease the reflection coefficient at its extremity and constitutes a simple line to the modulator top contact.

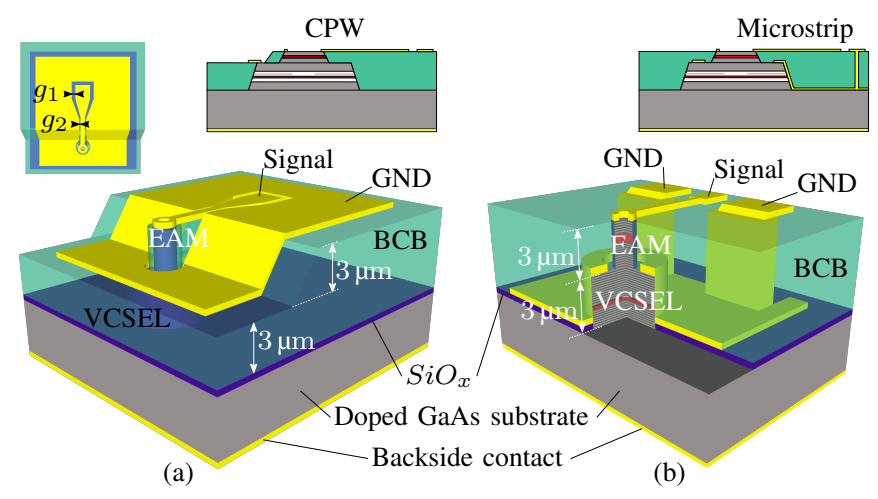

Fig. 3. (a) CPW and (b) MS designs for EAM-VCSEL device. 
The distances shown in Fig. 3a and Fig. 4a between the ground plane and the signal pad and line are named $g_{1}$ and $g_{2}$, respectively. It is necessary to bring the ground plane very close to the access line in order to confine the EM wave in the CPW structure and limit its interaction with the underlying substrate. With a BCB thickness of $6 \mu \mathrm{m}$, this confinement is verified using an EM simulator (ANSYS-HFSS) by setting $g_{1}$ and $g_{2}$ to $15 \mu \mathrm{m}$ and $5 \mu \mathrm{m}$, respectively. These last dimensions allow to decrease as much as possible the penetration depth of the magnetic field in the doped substrate while considering the fabrication constraints for the metal lift-off.

Furthermore, the BCB thickness is decreased to a few micrometers around the EAM mesa in order to reach the top VCSEL mesa and thus the intermediate contact. This last dimension is crucial and presents a very difficult process step since it is necessary to control the sidewall inclination to reach the VCSEL contact. Indeed, the VCSEL mesa diameter should not exceed $60-70 \mu \mathrm{m}$ to avoid problems related to long oxidation times in order to define the lateral confinement.

2) Process: Three different substrate-dopings have been used: n-doped, p-doped and Not Intentionally Doped (NID). Above the deposited BCB layer prepared as described in II-B, e-beam evaporated Ti/Au $50 / 200 \mathrm{~nm}$ bi-layer is used to form the pads.

3) Impact of the substrate doping with the CPW design: $\mathrm{CPW}$ thru structures formed by interconnecting the inner end of two EAM access lines, as shown in Fig. 4a, have been fabricated on the three substrate types and the aforementioned BCB layer. Measurements have been carried out using the same setup as in III-C. In order to understand the influence of substrate doping on the CPW access losses, the equivalent electrical $\Pi$-model shown in Fig. $4 \mathrm{~b}$ is extracted from the measured $S_{i j}$ data for NID, n and p-doped substrates. The values of $R_{s}, L_{s}, R_{p}$ and $C_{p}$ are plotted in Fig. 5. The CPW on the NID substrates exhibits a very small $R_{s}$ resistance around $1 \Omega$ below $60 \mathrm{GHz}, 2$ to 4 times lower than with doped substrates. With doped substrates, an important part of the return current goes through the substrate. The induced negative magnetic coupling increases the resistance $R_{s}$ and slightly lowers the inductance $L_{s}$. A highly doped substrate also acts as a poor metal layer that shields the CPW line and increases its equivalent capacitor $C_{p}$ compared to NID substrate. Undoped substrates are preferred in recent high-speed VCSELs for all these reasons [14]. Finally, the performance of CPW access lines is strongly dependent on the substrate conductivity. When a heavily doped substrate is used, the induced access resistance $R_{s}$ and capacitance $C_{p}$ are increased and may limit the maximum operating frequency of the modulator. This result motivated the study of a MS access line.

\section{B. MS access line}

The MS line can avoid the previously described substrateinduced effects if a ground plane supporting the return current is placed above the substrate. The electric contact required between the EAM and the VCSEL in order to apply bias voltage is used as a MS ground plane. In such way, the RF performance of this MS configuration becomes independent on

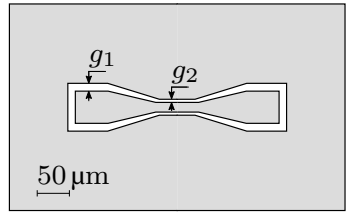

(a)

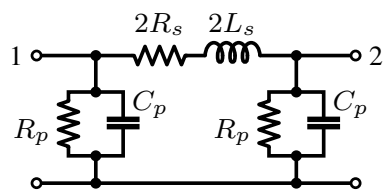

(b)
Fig. 4. (a) design of CPW thru device and (b) $\Pi$ equivalent lumped network used to model this device.

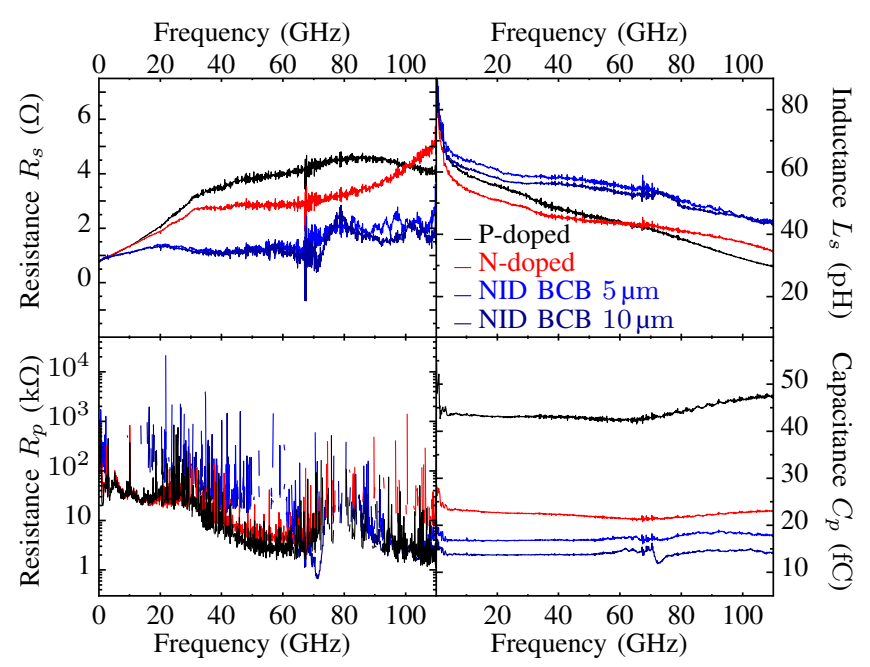

Fig. 5. Resistance $R_{s}$, inductance $L_{s}$, resistance $R_{p}$ and capacitance $C_{p}$ extracted from the CPW access measurements.

the substrate doping. Here, we compare it with the previously analyzed CPW access line. The shared ground contact is now moved under the $6 \mu \mathrm{m}$-thick BCB layer, while the access line remains on top of it. In such a way, the complicated angled photolithography step is no longer required, which substantially facilitates the fabrication process of the double mesa structure. As it is still necessary to have GSG contacts on the top of the chip to connect the RF probe, so the $\mathrm{BCB}$ layer is opened to bring the contacts to the ground pads as seen in Fig. 3b. The dimensions of this MS line design will be discussed in the next section.

We now compare in Fig. 6 the CPW and the MS access lines with the same lengths $(130 \mu \mathrm{m})$. Despite the losses brought by the substrate conductivity in the case of CPW access, the p-doped subtrate is used for this comparison since it minimizes the optical losses between the EAM and the VCSEL devices. The CPW and the MS line widths are 19 and $14 \mu \mathrm{m}$, respectively. The metal thickness is $0.3 \mu \mathrm{m}$ for the CPW. Thicknesses of $0.3 \mu \mathrm{m} / 0.3 \mu \mathrm{m}$ and $0.6 \mu \mathrm{m} / 0.7 \mu \mathrm{m}$ are studied for the signal/ground layers of the MS line.

In Fig. 6, the EM simulation data are in good agreement with our measurements on CPW. The CPW exhibits higher access resistance despite the larger line width because of the magnetically-induced substrate losses as previously explained. Doubling the MS line thickness has a very limited effect especially at the highest frequencies because of skin depth limitations. In both cases, the access inductance is comparable but the capacitance is much lower with a MS line thanks to its narrower signal line. Finally, even though the gain with the 


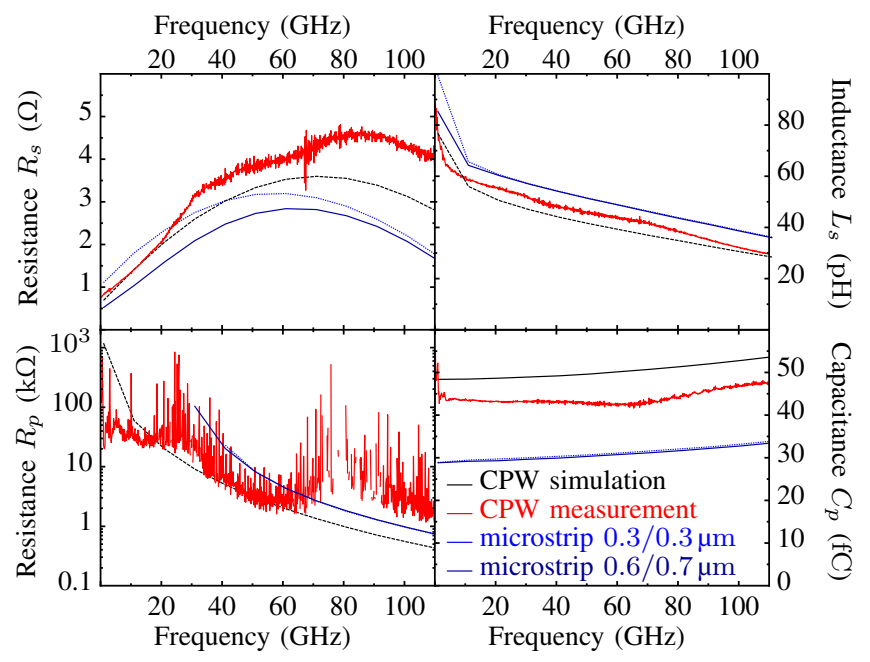

Fig. 6. $R_{s}, L_{s}, R_{p}$ and $C_{s}$ elements of measured (red) and simulated (black) CPW access compared to simulations of MS access with thin metal deposition (blue) and thicker metal (dark blue).

MS design is not so significant, this geometry is preferred due to an easier fabrication process.

\section{HIGH-FREQUENCY OPERATION OF THE EAM}

\section{A. Device description}

The GaAs-based modulator studied in this paper is composed of an undoped active region formed by multiple quantum wells (MQWs) playing the role of electro-absorber. The MQWs are embedded between two distributed Bragg reflectors (DBR) to provide an asymmetric Fabry-Perot structure. More details about the EAM structure can be found in [32]. No compositional grading is added at the DBR interfaces for the sake of simplicity of the epitaxial growth.

The modulation is achieved by applying an electric field perpendicular to the structure through quantum confined Stark effect (QCSE). QCSE modifies the band structure of the MQWs and thus the envelope function of the electrons and holes. In the case of the monolithic integration of the EAM onto a VCSEL, the VCSEL is $\mathrm{CW}$ powered by injecting current between the bottom electrical contact and the middle ground contact. The modulation frequency (RF) signal is applied between the top electrode of the EAM and the middle ground contact as shown in Fig. 3. The MS access line and the open vias to transfer the ground contact on top are presented in Fig. 3, too.

EAM samples are processed in the same way as for complete EAM-VCSEL devices, meaning that we fabricate the same double mesa structure to keep the same BCB thickness on the standalone EAM and on the EAM-VCSEL.

The operation of the standalone EAM device with a MS access line has already been demonstrated in [33], using a RF synthesiser source and a spectrum analyzer to measure the photodiode response. As shown in Fig. 7, modulation bandwidth above $28 \mathrm{GHz}$ has been achieved, limited by the photodiode responsivity.

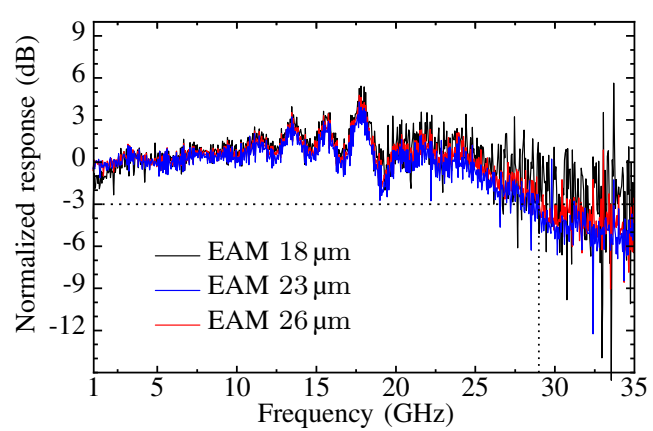

Fig. 7. Frequency modulation response of EAM devices of different diameters : $18 \mu \mathrm{m}$ (black), $23 \mu \mathrm{m}$ (blue) and $26 \mu \mathrm{m}$ (red).

\section{B. Equivalent circuit}

In order to further understand the EAM behavior at highfrequency, its electrical response is extracted from the measured reflection coefficient. The EAM is biased through 50 to $150 \mu \mathrm{m}$ MS lines terminated by GSG pads. To de-embed the signal pad and the access line contributions, two lines displaying the same geometrical characteristics but with different lengths are processed on the same wafer. The electrical characteristics of the signal pad and the line are recovered by using the method described in section III-B. Let $\boldsymbol{T}$ be the chain matrix comprizing the matrices $\boldsymbol{T}_{\boldsymbol{A}}$ of the pad and $\boldsymbol{T}_{\boldsymbol{l}}$ of the access transmission line

$$
\boldsymbol{T}=\boldsymbol{T}_{\boldsymbol{A}} \boldsymbol{T}_{\boldsymbol{l}}=\left(\begin{array}{ll}
t_{11} & t_{12} \\
t_{21} & t_{22}
\end{array}\right) .
$$

The de-embedded modulator impedance $Z_{m o d}$ is deduced from the measured impedance $Z_{\text {meas }}$ by using the expression

$$
Z_{\text {mod }}=\frac{t_{22} Z_{\text {meas }}-t_{12}}{t_{11}-t_{21} Z_{\text {meas }}}
$$

We are now able to get a better insight in the access element contributions to the overall impedance of the EAM. The EAM acts like a capacitance at low frequency, because of the $485 \mathrm{~nm}$-thick active region. Moreover, its impedance is more important for smaller diameter as seen in Fig. 8. At high frequency, the impedances of the structures with two different diameters tend to get closer to each other, which reflects a modification of the electrical behavior of the EAM. It can now be considered as a resistance in series with a capacitance more than a single capacitance.

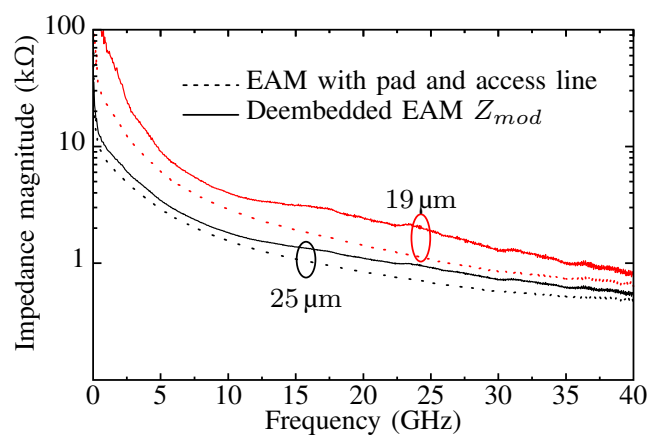

Fig. 8. Raw (---) and deembedded (-) EAM impedance $Z_{\text {mod }}$ for $19 \mu \mathrm{m}$ (red) and $25 \mu \mathrm{m}$ (black) devices. 
We can notice after the de-embedding, that the impedance of the EAM alone is higher than the one of the global circuit. Indeed, the presence of the pad and the line between the probe extremity and the modulator decreases the efficiency of the electrical injection in the device. The difference between the two curves, with and without de-embedding, is furthermore increased when the diameter is smaller (higher impedance) since the pad and line contributions, compared to the EAM impedance, are more important. This clearly emphasizes the relevance of the de-embedding process to know precisely the electrical behavior of the EAM.

\section{CONCLUSION}

In conclusion, thanks to an original and reliable planarization technological process, we have extracted a BCB permittivity of 2.56 up to $70 \mathrm{GHz}$ with a variation of $\pm 2.5 \%$ in this frequency range. Based on these characterizations, we designed two RF access geometries in order to optimize the high frequency modulation voltage actually applied to the EAM. Since working with doped substrate is mandatory for monolithically integrated EAM-VCSEL, CPW appears unsuitable due to the substrate-induced losses. It is shown that MS access line configuration makes easier the fabrication of a double mesa while being providing superior RF performances compared to CPW. Thanks to the method we developed, we have de-embedded the pad and the access line and extracted the impedance of the standalone EAM. This impedance could be drastically improved with DBR graded interfaces, in the same way as in a current-injected standard VCSEL. Finally, reducing further the EAM mesa diameter would considerably increase the electrical bandwidth of the EAM-VCSEL device.

\section{REFERENCES}

[1] C. C. Barron, C. J. Mahon et al., "Millimeter-wave asymmetric fabryperot modulators," IEEE J. Quantum Electron., vol. 31, no. 8, pp. 1484 1493, 1995.

[2] M. Zujewski, H. Thienpont, and K. Panajotov, "Electrical Design of High-Speed Electro-Optically Modulated Coupled-Cavity VCSELs," J. Lightwave Technol., vol. 29, no. 19, pp. 2992-2998, 2011.

[3] V. A. Shchukin, N. N. Ledentsov et al., "Digital data transmission using electro-optically modulated vertical-cavity surface-emitting laser with saturable absorber," Appl. Phys. Lett., vol. 104, no. 5, p. 051125, 2014.

[4] M. Yakimov, J. v. Eisden et al., "Concept of feedback-free highfrequency loss modulation in detuned duo-cavity vertical cavity surfaceemitting laser," J. Vac. Sci. Technol. B, vol. 28, no. 3, 2010.

[5] A. Paraskevopoulos, H.-J. Hensel et al., "Ultra-high-bandwidth (> $35 \mathrm{GHz}$ ) electrooptically-modulated VCSEL," in Optical Fiber Communication Conference, Anaheim, CA, USA, 2006.

[6] C. Chen and K. D. Choquette, "Multilevel Amplitude Modulation Using A Composite-Resonator Vertical-Cavity Laser," IEEE Photonics Technol. Lett., vol. 21, no. 15, pp. 1030-1032, 2009.

[7] P. E. Ganrou, W. B. Rogers et al., "Stress-buffer and passivation processes for $\mathrm{Si}$ and GaAs IC's and passive components using photosensitive BCB: Process technology and reliability data," IEEE Trans. Adv. Packag., vol. 22, no. 3, pp. 487-498, 1999.

[8] http://www.dow.com/cyclotene/solution/dielectric.htm.

[9] U. Dahlgren, J. Svedin et al., "An integrated millimeterwave BCB patch antenna HEMT receiver," in IEEE MTT-S International Microwave Symposium Digest, San Diego, CA, USA, 1994, pp. 661-664.

[10] H. Sakai, Y. Ota et al., "A millimeter-wave flip-chip IC using microbump bonding technology," in 1996 IEEE International Solid-State Circuits Conference, San Francisco, CA, USA, 1996, pp. 408-409.

[11] M. Janezic, D. Williams et al., "Permittivity characterization of low-k thin films from transmission-line measurements," IEEE Trans. Microw. Theory Techn., vol. 51, no. 1, pp. 132-136, 2003.
[12] S. Costanzo, I. Venneri, G. Massa, and A. Borgia, "Benzocyclobutene as Substrate Material for Planar Millimeter-Wave Structures: Dielectric Characterization and Application," J. Infrared, Millimeter, Terahertz Waves, 2009.

[13] A. N. AL-Omari and K. L. Lear, "Dielectric characteristics of spincoated dielectric films using on-wafer parallel-plate capacitors at microwave frequencies," IEEE Trans. Dielectr. Electr. Insul., vol. 12, no. 6, p. $11,2005$.

[14] A. Larsson, P. Westbergh et al., "High-speed VCSELs for short reach communication," Semicond. Sci. Technol., vol. 26, no. 1, 2011.

[15] P. Moser, J. A. Lott, and D. Bimberg, "Energy Efficiency of Directly Modulated Oxide-Confined High Bit Rate 850-nm VCSELs for Optical Interconnects," IEEE J. Sel. Top. Quantum Electron., vol. 19, no. 4, 2013.

[16] D. Burdeaux, P. Townsend, J. Carr, and P. Garrou, "Benzocyclobutene (BCB) dielectrics for the fabrication of high density, thin film multichip modules," J. Electron. Mater, vol. 19, no. 12, pp. 1357-1366, 1990.

[17] A. Modafe, N. Ghalichechian et al., "Embedded benzocyclobutene in silicon: An integrated fabrication process for electrical and thermal isolation in MEMS," Microelectron. Eng., vol. 82, pp. 154-167, 2005.

[18] W.-S. Shih, J. Yota, and K. Itchhaporia, "CON-TACT $®$ planarization process of spin-on dielectrics for device fabrication," J. Electrochem. Soc., vol. 6, no. 3, pp. 501-522, 2007.

[19] S. Abada, B. Reig et al., "Uniform fabrication of thick SU-8 patterns on small-sized wafers for micro-optics applications," in Micro-Optics 2014 vol. 9130. International Society for Optics and Photonics, 2014.

[20] "Reactive ion etching characteristics of partially-cured benzocyclobutene," in 15th Int. Conf. on Electronic Packaging Technology, author = Yang, Yang and Song, Zhen and Du, Yuxin and Wang, Zheyao, year $=2014$, pages $=862-866$, Chengdu, China

[21] H. V. Demir, J. F. Zheng et al., "Self-Aligning Planarization and Passivation for Integration Applications in III-V Semiconductor Devices," IEEE Trans. Semicond. Manuf., vol. 18, no. 1, pp. 182-189, 2005.

[22] L. Marigo-Lombart, J.-B. Doucet et al., "Self-aligned BCB planarization method for high-frequency signal injection in a VCSEL with an integrated modulator," in Semiconductor Lasers and Laser Dynamics VII, SPIE Photonics Europe, vol. 9892, Brussels, Belgium, 2016.

[23] W. B. Weir, "Automatic measurement of complex dielectric constant and permeability at microwave frequencies," Proc. IEEE, vol. 62, no. 1, pp. 33-36, 1974.

[24] A. Ghannam, C. Viallon, D. Bourrier, and T. Parra, "Dielectric microwave characterization of the SU-8 thick resin used in an above IC process," in 2009 European Microwave Conference (EuMC), Rome, Italy, 2009, pp. 1041-1044.

[25] J. Song, F. Ling et al., "A de-embedding technique for interconnects," in IEEE 10th Topical Meeting on Electrical Performance of Electronic Packaging, Cambridge, MA, USA, 2001, pp. 129-132.

[26] H. Ito and K. Masuy, "A simple through-only de-embedding method for on-wafer S-parameter measurements up to $110 \mathrm{GHz}$," in IEEE MTT-S International Microwave Symposium Digest, Atlanta, GA, USA, 2008, pp. 383-386.

[27] Y. Tretiakov, K. Vaed et al., "A new on-wafer de-embedding technique for on-chip rf transmission line interconnect characterization," in ARFTG 63rd Conference, Fort Worth, TX, USA, 2004, pp. 69-72.

[28] H. J. Saavedra-Gómez, J. R. Loo-Yau et al., "On-wafer CMOS transistors de-embedding method using two transmission lines of different lengths," in IEEE RFIC Symposium, Montreal, QC, Canada, 2012, pp. $417-420$.

[29] J. A. Reynoso-Hernández, C. F. Estrada-Maldonado et al., "Computation of the wave propagation constant gamma in broadband uniform millimeter wave transmission line," in 1999 Asia Pacific Microwave Conference, vol. 2, Singapore, 1999, pp. 266-269.

[30] I. J. Bahl and R. Garg, "Simple and accurate formulas for a microstrip with finite strip thickness," Proc. IEEE, vol. 65, no. 11, pp. 1611-1612, 1977.

[31] E. Hammerstad and O. Jensen, "Accurate Models for Microstrip Computer-Aided Design," in IEEE MTT-S International Microwave symposium Digest, Washington, DC, USA, 1980, pp. 407-409.

[32] L. Marigo-Lombart, S. Calvez et al., "Vertical electro-absorption modulator design and its integration in a VCSEL," J. Phys. D: Appl. Phys. vol. 51, no. 14, p. 145101, 2018.

[33] L. Marigo-Lombart, C. Viallon et al., "High frequency characterization of a vertical electro-absorption modulator for data communications," in IEEE International Topical Meeting on Microwave Photonics (MWP), Toulouse, France, 2018, pp. 1-4. 